American Journal of Infectious Diseases 8 (2): 79-91, 2012

ISSN 1553-6203

(C) 2012 Science Publications

\title{
Rendezvous with Tat: Transactivator of Transcription during Human Immunodeficiency Virus Pathogenesis
}

\author{
${ }^{1}$ Sneham Tiwari, ${ }^{2}$ Madhavan P.N. Nair and ${ }^{1}$ Shailendra K. Saxena \\ ${ }^{1}$ CSIR-Centre for Cellular and Molecular Biology, \\ Uppal Road, Hyderabad 500007 (AP), India \\ ${ }^{2}$ College of Medicine, Florida International University, Miami 33199 (FL), USA
}

\begin{abstract}
Acquired immune deficiency syndrome is a deadly disease caused by HIV, declared as a global catastrophe engulfing millions of lives per year. It took $\sim 1.8$ million lives in the year 2010 alone, as per reports of UNAIDS. HIV-1 is an enveloped retrovirus, having genome composed of two copies of ssRNA. It attacks the T-lymphocytes of the hosts, causes several multifaceted immune failures and finally leads to fatality. Our articles focuses on small regulatory tat (transactivator of transcription) gene which is responsible for the initiation and elongation of viral transcription through the LTR transactivation. It consists of two exons combining to form protein of 101 amino acids that enhances the efficiency of viral transcription. Tat exon 1(1-72) is important for viral transcription and Tat exon 2 (73-101) is important for cell adhesion and several activities like cellular uptake of the exogenous Tat, apoptosis, improvement of HIV-1 replication and IL-2 super induction in HIV-1-infected T cells. Tat plays vital role in LTR transcription, reverse transcriptions, Kaposi's sarcoma, neuropathogenesis, immunosuppression, concluding its importance. Eventually in future approaches, tat gene can be targeted for gene based drug targeting as Tat exon 1 is quite conserved and Tat exon 2 has important conserved motifs, which can behave as potent drug targets. Our review recommends to focus over tat gene, which might prove as a lead in HIV research, which should be treated with ssense of urgency.
\end{abstract}

Key words: Human Immunodeficiency Virus type 1 (HIV-1), Capsid (CA), Circulating Recombinant Form (CRF), Reverse Transcriptase (RT), neuropathogenesis

\section{INTRODUCTION}

The Human Immunodeficiency Virus type 1 (HIV1 ) is a deadly virus which is engulfing millions of lives every year globally. It has been reported that in 2010, $\sim 34$ million people are living with HIV. Over 7000 new HIV infections in a day happen according to the UN AIDS report of 2010. Of those around 97\% are from lower and middle income countries. About 1000 are children of under 15 years of age and 6000 are adults, out of which $48 \%$ are women. This is really an alarming point to be brought to the notice of world population. AIDS is engulfing human race with an extremely fast pace, which needs to be monitored and slowed down. The slow down process can be fueled up by gene based drug targeting which may help human race to combat with the virus.

The genome of the virus is a dimerized linear, positive-sense, single-stranded RNA, with each monomer of 7-13 kilobases $(\mathrm{kb})$ in size. The viral genomic RNA is present as a homodimer of two identical sequences; hence the viruses are diploid functionally. HIV encodes three polyprotein precursors (Gag, Pol, Env) which are required for the formation of infectious viral particles by infected cells. HIV-1 encodes for two regulatory proteins, which are required for viral gene expression at the transcriptional and posttranscriptional levels in infected cells (tat and rev) and accessory genes (vif, vpr, vpu and nef). HIV is enveloped virus, it have outer envelope which consists of gp120 (Surface glycoprotein) and gp41 (transmembrane glycoprotein). Both help in attachment and recognition to cells to entry. Other structural proteins are Matrix (MA) p24 and Capsid (CA) p17. HIV-1 Tat is a small protein that localizes to the nucleus of infected cells, acting as a potent transcriptional activator that is required for the synthesis of the full length viral RNA. The HIV-1 Tat protein plays a central role in the regulation of HIV-1 gene expression both at the level of transcription and protein synthesis.HIV is a member of the lentivirus subfamily of retroviruses that produces chronic infection in the host and gradually damages the host's immune system. HIV-1 is the predominant type in the

Corresponding Author: Shailendra K. Saxena, CSIR-Centre for Cellular and Molecular Biology, Uppal Road, Hyderabad 500007 (AP), India 
world. On the basis of genetic similarities, the virus strains can be distinguishly classified into types, groups and subtypes. The virus is divided into type $1 \mathrm{HIV}-1$ and HIV-2 based on genetic variations. Worldwide, the predominant virus is HIV-1, the strains of HIV-1 can be classified into three groups: the "major" group $\mathrm{M}$, the "outlier" group $\mathrm{O}$ and new group, $\mathrm{N}$ (non-M/non-O). Group $\mathrm{M}$ is the most important, being widely distributed in the world. Group $\mathrm{M}$ is divided into nine subtypes, A to K. Moreover, these subtypes recombine to generate a Circulating Recombinant Form (CRF) that is considered to be a new subtype. HIV-1 primarily infects CD4+ T-lymphocytes and monocytes/macrophages, but also astrocytes and cells of the central nervous system (brain microglial cells) are targets. Virus replication leads to a slow and progressive destruction of the immune system. HIV-1 makes use of the replication machinery of the host cell, which minimizes the number of potential viral targets. HIV has genetic blueprint made of Ribonucleic Acid (RNA) instead of Deoxyribonucleic Acid (DNA) and replicates through reverse transcription. HIV is enveloped virus, it have outer envelope which consists of gp120 (Surface glycoprotein) and gp41 (transmembrane glycoprotein). Both help in attachment $\&$ recognition to cells to entry. Other structural proteins are Matrix (MA) p24 and Capsid (CA) p17. Outer lipid membrane is derived from cells during the process of viral budding, enzymes involved are: Integrase (IN), Reverse Transcriptase (RT) and Protease. The genome of the virus is a dimerized linear, positive-sense, singlestranded RNA, with each monomer of 7-13 kilobases $(\mathrm{kb})$ in size. The viral genomic RNA is present as a homodimer of two identical sequences; hence the viruses are diploid functionally. HIV-1 genome encodes for three structural genes ( $g a g, p o l$ and $e n v$ ), regulatory genes (tat and rev) and accessory genes (vif, vpr, vpu and nef). HIV-1 primarily infects CD4+ T-lymphocytes and monocytes/macrophages, but also astrocytes and cells of the central nervous system (brain microglial cells) are targets. Virus replication leads to a slow and progressive destruction of the immune system. HIV-1 makes use of the replication machinery of the host cell, which minimizes the number of potential viral targets (Schiralli and Henderson, 2012).

HIV/AIDS epidemiology: HIV/AIDS is declared as a global catastrophe which is affecting millions of lives and is proving as major health hazard. According to 2010 reports on the global AIDS epidemic, this global epidemic is a major concern today as approximately 38 million people globally are living with HIV/AIDS and more than 4.1 million people acquired new infection after 2005 and around 2.8 million people lost their precious lives to AIDS. Globally, approximately than 25 million people with HIV died since the pandemic began, out which approximately 50 lakhs of population belongs to United States. 1990s proved to be a major peak decade which reported maximum infection rates. HIV/AIDS has emerged as a health and development hazard over the past decade and the disease has crept to every corner of the world and is still rapidly growing. The numbers of people living with HIV have continued to rise, due to population growth and, more recently, the life-prolonging effects of antiretroviral therapy. In subSaharan Africa, the region with the largest burden of the AIDS epidemic, data also indicate that the HIV incidence rate has peaked in most countries. HIV prevalence has been reported to be declining in four states in India, including Tamil Nadu, where prevention efforts were scaled up in the late 1990s. However, HIV prevalence is increasing in some countries, China, Indonesia, Papua New Guinea and Viet Nam and also there are signs of HIV outbreaks in Bangladesh and Pakistan and Africa remain the global epicenter of the AIDS pandemic. HIV subverts the working of human immune system which in turn makes infected people more susceptible to various infectious diseases. The most devastating result of HIV infection is AIDS which is one of the most serious health hazards in the world till date. The first HIV case was identified in 1981 and since then AIDS has engulfed millions of lives which marks a terrible loss on Indian and global health status economy. Despite advances in HIV/AIDS research, the pandemic continues to undermine lives, communities and societies. There is no cure for AIDS or a vaccine to prevent infection. Scientific, medical, logistical and operational challenges remain to make HIV therapies, prevention services and other interventions available to poor countries. If left unchecked, HIV/AIDS will continue to have devastating consequences around the world for decades to come in every sector of society.

Tat (transactivator of transcription): a vital regulatory gene: Tat is a regulatory gene which encodes for the Tat protein, which is a small protein of 101 amino acids. Amino acids from 1-72 belong to Tat exon 1 and 72-101 belong to Tat exon 2 (Debaisieux et al., 2012). Tat exon 1 plays a vital role in viral transcription and Tat exon 2, which was initially considered to be non essential, is now said to be of great importance in cell adhesion and indirectly essential for viral transcription and cell apoptosis Cytoskeleton-related functions as cell morphology, proliferation, chemotaxis, polarization and actin polymerization are studied to be deeply altered in 
absence of Tat exon 2. There are emerging findings that the Tat exon 2 is important for optimal transactivation and virus replication. Two short motifs in Tat exon 2 have great functional importance. The first is RGD motif that is used as a cell adhesion signal for binding to cellular integrins (Brake et al., 1990). This RGD motif however not found in HIV-2 or SIV is the important protein portion for cellular uptake. Second is ESKKKVE motif which is conserved in most HIV-1 Tat protein and is partially preserved in HIV-2 and SIV Tat. It also plays extracellular role in increasing AIDS pathogenesis and progression. Among the HIV regulatory genes, Tat has a fundamental function in HIV pathogenesis and disease progression. If Tat is absent the virus may still be able to infect the cell, but it will not replicate. Tat is produced very early after HIV infection, even before the virus integrates into the host cell's genetic machinery. Moreover, Tat is necessary for the virus to replicate within the host cells and also to infect naive cells as acutely infected cells have the potential of releasing Tat and it leads to recruitment and activation of other uninfected cells. This provides new cells for the virus to infect, helping to spread the HIV infection throughout the body in a very fast pace. Tat plays many important roles and has vital functions in HIV pathogenesis, which are covered briefly in the article. Tat is a major viral transactivator required for HIV-1 replication. In the nucleus Tat greatly stimulates the synthesis of full-length transcripts from the HIV-1 promoter by causing efficient transcriptional elongation. Tat induces elongation by directly interacting with the bulge of the Transactivation Response (TAR) RNA, a hairpin-loop located at the 5'end of all nascent viral transcripts and by recruiting cellular transcriptional co-activators. Tat binds to a short nascent stem bulge loop leader RNA (TAR), consisting of base paired stem, a small (trinucleotide) non base paired bulge and a 6-nucleotide Guanine rich loop. Tat interacts with a 59-nucleotide stem-loop TAR RNA and hence transactivates the viral LTR-promoter and Tat also recruits the positive transcription elongation complex-b (pTEF-b) (Tahirov et al., 2010), which consists of CDK9 and cyclin T1 that hyperphosphorylates the C-Terminal Domain (CTD) of RNA polymerase II, which has a very vital role in enhancing viral transcription (Jager et al., 2011). Inhibition of P-TEFb activity is governed by Hexim1 that act on Cdk9 and Cyclin T1. HIV-1 Tat/TAR complex abolish Hexim1 inhibition, helping in transcription of viral genes (Czudnochowski et al.,
2012). Tat can very easily be translocated across the cell lipid membrane, its mechanism is studies via NMR studies by researchers, which makes it extremely dangerous and it help in intensifying the viral pathogenesis (Herce and Garcia, 2007). The error prone methodology of HIV-1 replication results in varied viral sequences, but Tat can very intelligently retain its complex functions in viral gene expression and replication, mechanism to which is still unknown. Important amino acid positions in Tat are conserved and it also conserves the correlated pairs which have important role in functioning of Tat (Dey et al., 2012). Human Endogenous Retroviruses (HERVs) constitute $8 \%$ of human genome and recent studies have demonstrated that Tat plays a vital role in setting off expression of HERV-K (HML-2) in the intensifying infection (Gonzalez-Hernandez et al., 2012). Recent finding also point towards the role of Tat in neuropathogenesis, as reports state that endolysosomes take active involvement in HIV-1 Tat induced neurotoxicity posing as target for therapeutics against HAND (Hui et al., 2012).

Tat structure: Tat encodes a protein of 101 amino acids (Fig. 1), which is encoded by two exons; exon 1 and exon 2 separated by huge intron (López-Huertas et al., 2010). The Tat protein has six functional domains (acidic, cysteine rich, core, basic, glutamine rich and RGD motif). Highly conserved regions within the domains of tat gene regulate its functions. But the Tat protein of the different HIV subtypes shows variations in these amino acid sequences. Amino acids forming Tat exon 1 are highly conserved and comprise five regions that are needed for transactivation of the viral LTR promoter. The first three regions constitute a minimal activation domain capable of binding to cyclin T1. The combined results from many laboratories have permitted an arbitrary demarcation of "domains" (or functional region) in Tat protein (Kuppuswamy et al., 1989).

Tat exon 1: HIV-1 Tat exon 1 comprises of encodes for 71 amino acids. This exon is divided into five domains, which are named according to their composition.

Domain 1: acidic (1-21 aa): The N-terminus of Tat protein is a proline rich region called acidic region and has a conserved Trp11 residue. Mutation in this region can be tolerated by virus without causing any major change without loss of biological activity. 1-20 aa is also called as neutralization antibody epitope of Tat protein. 
Am. J. Infect. Dis., 8 (2): 79-91, 2012

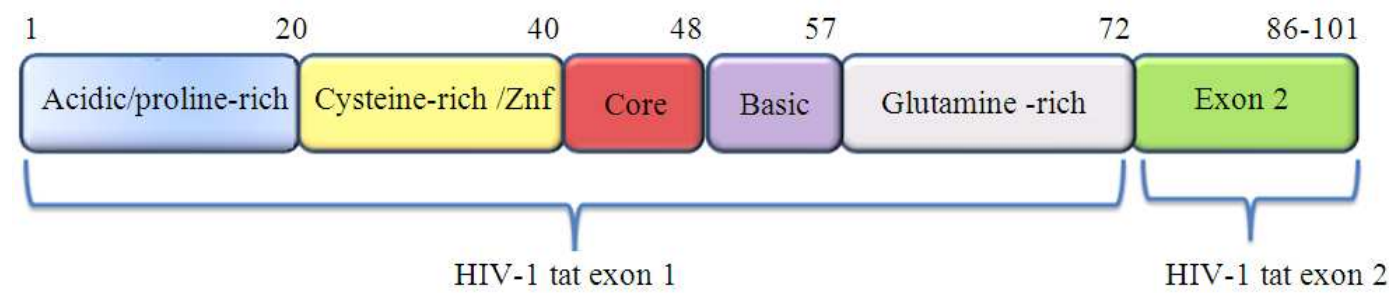

Fig. 1: Diagrammatic representation of HIV-1 Tat: Tat exon 1: Domain 1: Acidic/Proline-rich region, Domain 2: Cysteine-rich region. Domain 3: Core region, Domain 4: Basic region, Domain 5: Glutamine-rich region; followed by Tat exon 2

Domain 2: cysteine rich (22-37 aa): The cysteine rich region contain seven conserved cysteines amino acid at positions $22,23,27,30,31,34$ and 37 and these are also highly conserved between different isolates of HIV-1s (except subtype $\mathrm{C}$ have C31S variation). Individual mutations in six of the seven cysteines (except C31S) may abolish regular function of Tat. These cysteines appear to be free and they are also proposed as a metal chelating (bound to Zinc) dimerization domains (Frankel et al., 1988). This region has also been showed to be used for intra-molecular disulfide bond formation in monomeric molecule of Tat protein found inside cell (Koken et al., 1994). The cysteine residues at position 31 are critical for binding to the NMDA receptor on neurons which increase neurotoxicity (Li et al., 2008):

Domain 3: core (38-48 aa): This domain contains a RKGLGI motif that is conserved between HIV-1, HIV2 and SIV Tat. This region, in conjunction with the amino terminus and cysteine rich domain, has been suggested to confine to the minimal activation domain of HIV-1 Tat (Derse et al., 1991). Also called as central domain, it contains the conserved 36VCFT39 motif involved in tubulin binding and apoptosis and the 41KGLGI45 motif that constitutes the minimal activation domain:

Domain 4: TAR binding basic (49-59 aa): This domain contains a basic RKKRRQRRR motif. These amino acids are important for TAR-RNA binding properties of Tat and are important for nuclear localization of the protein (Ruben et al., 1989). Recent studies shows that this short domain may be insufficient in determining the entire specificity of Tat-TAR binding since amino acids outside of the basic domain also contribute to this interaction (Graeble et al., 1993). This domain contains the nuclear localization signal 49RKKRRQRRR57 necessary for binding to TAR and also this region is the minimal neurotoxic region causing cell death.
Domain 5: glutamine rich region $(60-72$ aa): This domain also has a very vital role as amino acids of this domain are conserved and involved in Tat mediated apoptosis of T-cells, leading to infection and disease progression, supporting healthy stay of virus in the host.

Tat exon 2: Tat exon 2(73-101 aa) is the region which contains two main motifs: A 78RGD80 sequence involved in cell adhesion and the 86ESKKKVE92 motif important for NF-kB transactivation. Tat exon 2 has been involved in several activities as the cellular uptake of the exogenous Tat, apoptosis through increasing activity of caspase-8, improvement of HIV-1 replication in tissue culture and primary PBLs and IL-2 superinduction in HIV-1-infected $\mathrm{T}$ cells after CD3/CD28 co-stimulation. Both extra and intracellular Tat display the same structure, but this protein acts differently when is synthesized inside the cell during HIV-1 infection than when is released from infected cells. Intracellular Tat is able to protect cells from apoptosis (Stauber and Pavlakis, 1998), whereas extracellular Tat is considered a pro-apoptotic factor that contributes to AIDS-associated immunosuppression and pathologies as Kaposi's sarcoma, dementia and degenerative diseases. One proapoptotic mechanism described for extracellular Tat involves its attachment to microtubules and to the actin cytoskeleton, altering the cytoskeleton dynamics. Cytoskeleton is involved in cell motility, proliferation and intracellular trafficking, as well as in cell shape maintenance and polarity. Actin filaments, actinbinding proteins and microtubules are not only essential for regulating cell death, but also play a major role in HIV-1 infection. Deregulation of gene expression was 4-fold higher in T cells expressing intracellular Tat 101 than Tat truncated at Tat exon 2 (Tat72).

To check upon the efficiency of Tat, a mutant infectious clone was engineered by introducing a stop codon at amino acid 72 in the Tat open reading frame. It was seen that this mutant replicated very slowly as 
compared to the wild-type in peripheral blood lymphocytes (Mayol et al., 2007). Studies have suggested a critical role for the glutamic acids at positions 92, 94 and 96 or lysines at positions 88, 89 and 90, present in Tat exon 2 in activating NF-kB, transactivating the HIV-1 LTR and enhancing HIV-1 replication in $\mathrm{T}$ cells (Mahlknecht et al., 2008). Cytoskeleton-related functions as cell morphology, proliferation, chemotaxis, polarization and actin polymerization were studied to be deeply altered in Jurkat Tat 101, but not in Jurkat-Tat 72 and expression of cell surface receptors was impaired by intracellular Tat101 but not by Tat 72 . These all studies show that these modifications were greatly dependent on Tat exon 2 (López-Huertas et al., 2010).

HIV-1 TAT functions: Primary role for Tat is in regulating transcription from the HIV-1 promoter region termed the LTR. Tat also has other major roles to play. Tat has intense effect on both HIV-1-infected and uninfected cells and actively contributes to the pathology of AIDS. For example; Kaposi's sarcoma lesions are caused by the direct interaction of Tat with a basic Fibroblast Growth Factor (bFGF), Tat inhibits the proliferation of uninfected $\mathrm{T}$ cells by the repression of MHC-1transcription and contributes to the dementia associated with AIDS. Tat induces an immunosuppressive effect on uninfected macrophages by increasing the expression of Fas ligand. Extracellular Tat regulates the expression of the HIV-1 co-receptor CXC-chemokine receptor 4 on T-lymphocytes (CXCR4). During HIV infection transcripts of Tat are found before integration and lead to increased $\mathrm{T}$ lymphocyte activation and viral replication. Tat is secreted by infected cells and is taken up by bystander cells in which Tat may either reactivate transcription or may exert its harmful functions on cellular mechanisms. Other functions have also been attributed to Tat as enhancing viral reverse transcription, mimicking chemokine functions, suppressing antigen specific CD8+ T-cell immune response and thereby upsetting the metabolism of host cells. The effect of Tat increases by several folds, if it is accompanied by some drugs of abuse like methamphetamine, cocaine, alcohol, which intensify the potential of Tat (Wires et al., 2012). Tat increases the HIV-1 RNA translation rate (Charnay et al., 2009).

Effect of Tat on neurons: Tat induces striking increases in levels of intracellular calcium in neurons. Initially there is a rush of intracellular calcium followed by prolonged increase in concentration of cytoplasmic calcium resulting from an influx of extracellular calcium (Haughey et al., 1999). This gradually leads towards mitochondrial calcium uptake, inhibition of electron transport chain, generation of reactive oxygen species, activation of caspases and finally results in apoptosis (Kruman et al., 1998; New et al., 1997; Norman et al., 2007). Tat has an ability to mediate neuronal dysfunction by inducing neuronal aggregation and combining neuritic processes, which is a vital function of RGD (arginine-glycine-aspartic acid) sequence present in Tat exon 2 (Kolson et al., 1993). Tat is also efficient in causing release of neurotoxic substances from glial cells and macrophages (Nath et al., 1996). Tat stimulates the production of proinflammatory cytokines (Pulliam et al., 2007) and neurotoxins in the brain cells. Tat induces many cytokines and chemokines in macrophages and astrocytes (D'Aversa et al., 2004) importantly like tumor necrosis factor-a (TNF- $\alpha)$, monocyte chemo attractant factor-1 (MCP-1)/CCL-2 and CXCL-10 (Eugenin et al., 2005). MCP-1/CCL-2 is a highly potent chemoattractant for monocytes. Levels of this chemokine are elevated in the CSF and brain of patients with HIV dementia. Interestingly, MCP-1/CCL-2 may protect neurons against Tat-induced neurotoxicity suggesting that this chemokine may act as a doubleedged sword (Buonaguro et al., 1992).

Tat as a chemoattractant: Besides the ability to induce the chemokine production, Tat may also have some chemoattractant properties for monocytes (ElHage et al., 2006). Studies on significant sequence homology have shown to be having similarity with key residues of functional importance in chemokines helping in their potency to attract monocytes. Crossdesensitization studies have indicated that Tat shares receptors with $\mathrm{MCP}-1, \mathrm{MCP}-3$ and eotaxin, also $\mathrm{CCF}$ motif, SYXR motif. The greatest similarity is noted with the MCP/CCL family of chemokines. Any mutations in $\mathrm{CC}$ motif of Tat may impair its chemotactic properties (Ranga et al., 2004).

LTR transcription: The HIV-1 LTR serves as the site of transcriptional initiation and harbours cis-acting elements required for RNA synthesis. The LTR is composed of three regions: U3 (for unique, 3' end), R (for repeated) and U5 (for unique, 5' end). Transcription initiates at the U3/ R junction. Tat protein is expressed initially to regulate the LTR transcription, it transactivate the transcription of genome at elongation stage. In absence of Tat transcription initiation is completed efficiently but aborted at elongation stage consequently giving short fragment of transcript but in presence of Tat protein dramatically increases the level of complete transcripts (Kessler and Mathews, 1992). Principal function of Tat is the transactivation of the HIV LTR. Tat also transactivates 
several cellular genes and it has been demonstrated that Tat plays a critical function in cytopathogenicity that is independent of HIV LTR Transactivation.

Tat is required for HIV reverse transcription: Studies have shown that Tat is able to fuel up efficient reverse transcription. Tat binding to TAR RNA may alter TAR structure so that the initiation of reverse transcription is enhanced. Mutations in Tat gene genuinely decrease the initiation of reverse transcription of HIV-1 replication by 1000 folds. Viruses which lack Tat are seen to be defective in endogenous assays of reverse transcription, even though they have same levels of reverse transcriptase. These studies highlight that Tat protein, is important for efficient HIV-1 reverse transcription.

Role in Kaposi's sarcoma: The extra-cellular role assumed for Tat is its indirect contribution to Kaposi's Sarcoma (KS) associated with AIDS. KS is angiogenic lesion, an unusual neoplasm caused by the human herpesvirus-8 (HHV-8), which affects the skin of elderly males characterized by new blood vessel formation, an inflammatory infiltrate and proliferation of a spindle-shaped cell population which is considered to be the "tumor" cell population of the lesion. However, AIDS-related KS (AIDS-KS) is more dangerous. Tat induces the growth of primary endothelial cells activated with inflammatory cytokines (Ensoli et al., 1989) like interferon (IFN)- $\gamma$, TNF and IL-1 $\beta$, which are increased in the blood and lesions of AIDS-KS individuals which in turn supplement the synthesis and release of basic Fibroblast Growth Factor (bFGF). Tat competes with bFGF for heparin-binding sites, increasing soluble bFGF to high concentrations which promote endothelial growth and upregulates the integrins $\alpha 5 \beta 1$ and $\alpha v \beta 3$ receptors for fibronectin and vitronectin, respectively, both of which are highly expressed in AIDS-KS. One of the similarities between fibronectin, vitronectin and subtypes B and D Tat is the presence in the C-terminal domain of Tat of an RGD motif, which represents the principal cell attachment moiety. Based on these observations, it was proposed that the Tat protein, in combination with a cytokine imbalance, could be a causative agent for AIDS-KS Several groups began to investigate the mechanism for the angiogenic and tumorigenic effects of Tat (Douglas et al., 2007; Courter et al., 2010).

Cell apoptosis: Tat disturbs cellular metabolism by inhibiting antigen-specific $\mathrm{T}$ lymphocytes proliferation and causing apoptosis. Distortion of microtubules polymerization causes Tat-induced apoptosis of T-cells. LIS1 is a microtubule-associated protein that facilitates microtubule polymerization which involves apoptosis, autophagy and activation-induced cell death. Tat performs the induction of apoptosis through BIM (a pro-apoptotic protein of the Bcl-2 family), that provokes Bcl-2 anti-apoptotic proteins. Even though Tat protein has been reported as a growth factor and protector of transfected cell lines against apoptosis (Zauli et al., 1995) but in contrast, it has also been shown that, Tat in addition to the inhibition of T-cell responses to antigens, also increases the apoptotic rate of T-cells. Tat has been consistently found to upregulate the expression of CD95-fas (Westendorp et al., 1994; Li et al., 1997). Tat-transfected Jurkat cells cultured in low serum show an increased level of apoptosis, during experimentation.

Extracellular activity of tat: Tat can easily take an exit from transfected cells in the absence of apoptosis (Westendorp et al., 1995) showed that Tat is released from HIV-infected cells at substantial levels in HIV patients. These serum levels are similar to that of many cytokines having several functions. Extracellular Tat may directly affect HIV replication in vivo and in vitro. Tat is released from HIV-1-infected cells. Extracellular Tat has the ability to enter the cell and nucleus in an active form and to stimulate the transcriptional activity of HIV-LTR. Extracellular Tat induces many biological effects on target-uninfected cells.

Tat and integrins: The sequence of the Tat exon 2 contains an RGD motif in the C-terminal domain, which is also the key sequence of many extracellular matrix proteins like fibronectin and vitronectin that is recognized by their receptors of integrin family involved in cell adhesion. Brake et al. (1990) showed that the RGD sequence of Tat could mediate cell adhesion (Ruoslahti, 1996). Several studies indicate that integrins can recognize the Tat protein as a substrate. Even the basic domain of Tat is also involved in integrin-mediated cell adhesion (Vogel et al., 1993). Tat-integrin binding exacerbates integrin-extracellular matrix ligand interactions.

Tat and immunosuppression: The immune suppression seen with AIDS appears to affect cells that are not infected with HIV. Several studies have shown that there is immunosuppression of non-HIV-infected cells from AIDS patients and that the number of immunosuppressed cells substantially exceeds that of the potentially HIV-infected cells. Tat proteins released from HIV-infected cells mediate such immune suppression. Tat has been linked to induction of Tcell anergy, T-cell apoptosis and to a T-cell hyperactivation which appears to prime cells for infection by HIV. 
Tat and HIV infection: Partially activated $\mathrm{T}$ cells show increased rate of apoptosis, resulting from an incomplete stimulation through $\mathrm{T}$-cell receptor. Tat is capable of partial, Tcell activation. HIV does not readily infect resting $\mathrm{T}$ cells, rather $\mathrm{T}$-cell activation is a requisite for HIV infection and it will have detrimental effect to the host immune response as well. This role is fulfilled by Tat. HIV-infected cells appear to be hypersensitive to $\mathrm{CD} 3 / \mathrm{CD} 28$ costimulation, with an increased production of IL-2. Transfection with Tat has been shown to increase IL-2 production. The observation of partial T-cell activation by Tat suggested that stimulation by extracellular Tat may result in improved HIV infection.

Tat in neuropathogenesis: Tat regulatory protein actively releases from the cell and interacts with cell surface receptors of other uninfected cells in the brain leading to cellular dysfunction. The Tat protein is highly potent and has the unique ability to travel along neuronal pathways. Tat protein has central role in the neuropathogenesis of the HIV infection. Tat stimulates, Lipoprotein Receptor related Protein (LRP), postsynaptic density protein-95 (PSD-95) and the Nmethyl-D-aspartic acid (NMDA) receptors form a macromolecular complex in neurons which leads to neuronal injury. The core domain of Tat is also involved in interaction with LRP domains II, III and IV. Tat can also bind directly to the NMDA receptor through Cys-Cys interactions with the extracellular domain of the NR-1 subunit (Li et al., 2009) leading to further neuropathogenesis.

HIV Tat protein has a role in cell apoptosis and growth impairment: HIV-1 Tat protein has extracellular activities and affects surrounding cells of immune systems to increase complexity of HIV pathogenesis. Tat protein affects the cells by interacting to cell growth regulatory protein, which lead to impairment to cell functions and resulting in cell apoptosis or tumor formation. Cyclin B1, an important cell cycle regulator, was up-regulated in lymphocytes of Human Immunodeficiency Virus (HIV)-infected patients. It is showed that Tat protein regulates cyclin B1 levels in two different ways: first, Tat stimulates the transcription of cyclin B1, which increases cyclin B1 levels and promotes the cells apoptosis; and secondly, Tat stimulates polyubiquitination-mediated degradation of cyclin B1 through binding to the $\mathrm{N}$-terminal of cyclin B1 (aa 61-129) hence Tat-regulating cyclin B1 affects the status of HIV. Tat stimulates cyclin B1 expression to slow down the host cell cycle progress and to promote the host cell apoptosis, which might facilitate HIV release (Zhang et al., 2010).
RGD domain of Tat exon 2 is known to interact with cell surface adhesions protein integrins by mimicking it's natural ligand i.e., fibronectin, which also have RGD domain, RGD motif is crucial to interact with integrins and consequently entering to cell and also promote various signal pathways. Recently RGD motif is also found in a cellular regulatory protein named Osteopontin. Osteopontin (OPN) is a phosphoglycoprotein that has important physiological functions in bone remodeling, immune response and inflammation. It is also a tumor-associated protein, which mediates tumor transformation and malignant progression. Elevated OPN levels have been shown to correlate with increased tumor progression and poor survival in many solid tumors. RGD plays a major role in facilitating the regulatory function of OPN protein, it is observed that OPN suppress the apoptosis in tumors in RGD dependently, The RGD Domain of OPN Mediates Apoptosis Partially via NF-kB Activation.

The importance of tat exon 2: Tat exon 2 has been less studied. However, several recent findings from HIV-2 and SIV Tat show, that Tat exon 2 contributes towards optimal transactivation indirectly. There are emerging findings that Tat exon 2 is important for transactivation, transrepression (Howcroft et al., 1993) and virus replication (Neuveut et al., 2003). Two short motifs in the Tat exon 2 of HIV-1 Tat have extreme functional importance. The first is RGD motif that is used as a cell adhesion signal for binding to cellular integrins (Brake et al., 1990) and other is ESKKKVE motif which is conserved in most HIV-1 Tat protein and has been described as critical for NF-kB transactivation.

RGD motif: Tat exon 2 encodes a 29 amino-acid Cterminal sequence that is not required for transactivation but does contain an RGD motif, which is important in binding to $\alpha_{\mathrm{v}} \beta_{3}$ and $\alpha_{5} \beta_{1}$ integrins (Fig. 2). Tat has an unusual property for a transcription factor; it can be released and enter cells freely, retaining its activity, enabling it to up-regulate a number of genes. The HIV-1 Tat protein activates transcription of the viral LTR promoter through interaction with the nuclear transcription machinery of the host cell. Tat can also activate the LTR promoter in inter-cellular manner whose mechanism is yet not fully understood, but many studies suggest that may be Tat protein itself is secreted by cells and taken up by other cells. According to this mechanism, inter-cellular transcriptional activation by Tat should be very similar to intra-cellular trans-activation. A large number of cytokine genes were recently reported to be Tat-responsive, raising the possibility that such cytokines are involved in inter-cellular Tat action. 
Am. J. Infect. Dis., 8 (2): 79-91, 2012

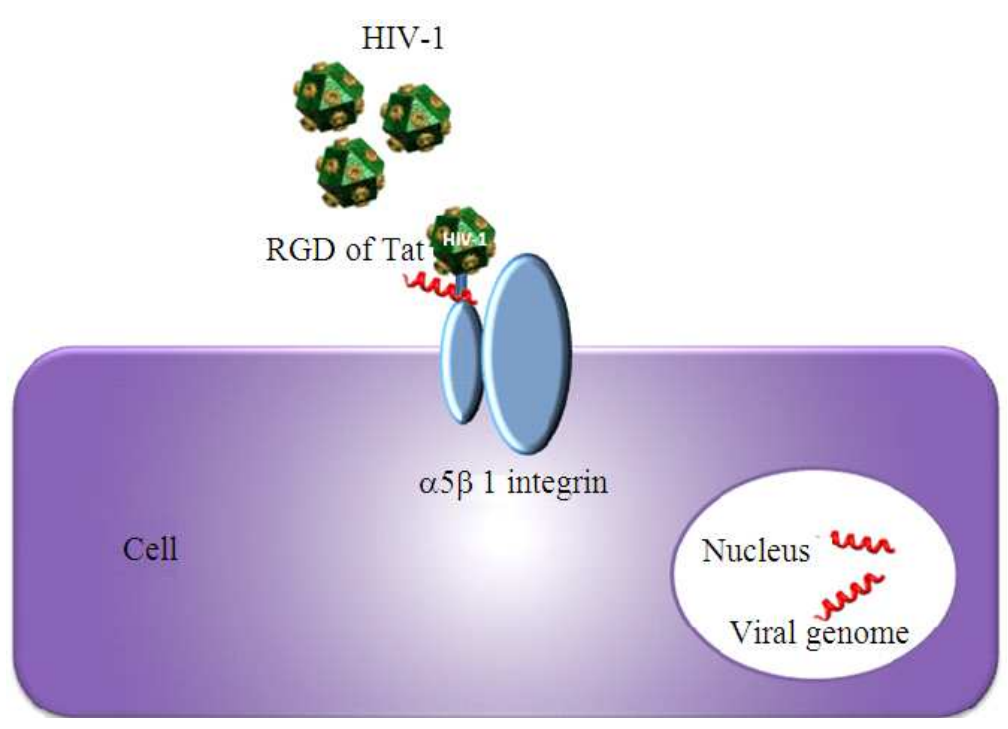

Fig. 2: RGD motif of Tat exon 2 is important for cell adhesion via binding to $\alpha_{\vee} \beta_{3}$ and $\alpha_{5} \beta_{1}$ integrins

Studies suggest that Tat protein itself is exported and transported into the nucleus of another cell and Tat exon 2, having the RGD motif probably interacts with an integrin receptor, required for cellular uptake of the Tat protein (Verhoef et al., 1996).

The 86ESKKKVE92 motif: There is important contribution of the extreme $\mathrm{C}$ terminus of HIV-1 Tat to maximize nuclear transcription factor NF-kB activation, Long Terminal Repeat (LTR) transactivation and viral replication in T cells. Several studies have been done to check the importance of the extreme C-terminal of the Tat gene, so many C-terminal deletion and substitution mutants were made with the infectious clone of HIV strains (not having Tat exon 2) and were assayed for their ability to transactivate NF-kB-secreted alkaline phosphatase and HIV-1 LTR-luciferase reporter constructs for low concentrations of Tat. A mutant infectious clone of HIV was engineered by introducing a stop codon at aa 72 in the Tat open reading frame (not having Tat exon 2) replicated at a significantly lower rate than the wild-type in primary peripheral blood lymphocytes. So studies have suggested a critical role for the glutamic acids at positions 92, 94 and 96 or lysines at positions 88,89 and 90 , present in the Tat exon 2 in activating NF-kB, transactivating the HIV-1 LTR and enhancing HIV-1 replication in T cells (Mahlknecht et al., 2008).

HIV Tat proteins are specified by two coding exons. Full-length HIV-1 Tat has been shown to have several roles, including the modulation of host gene expression activation of resting $\mathrm{T}$ cells, (induction of chemokine production (Izmailova et al., 2003) and direct suppression of antigen-specific CD8 $\mathrm{T}$ cell immune response in vivo. Tat exon 2 is shown to be involved in the cellular uptake of the exogenous Tat protein (Ma and Nath, 1997). The human translation elongation factor 1 binds to Tat exon 2, resulting in a frequent decrease of the efficiency of the translation of cellular mRNAs (Xiao et al., 1998). Tat induction of apoptosis requires expression of Tat exon 2 and is associated with the increased activity of caspase-8 (Bartz and Emerman, 1999). In lymphoid CD4 T cells, Tat-mediated activation of cJun $\mathrm{N}$ Terminal Kinase (JNK) requires Tat exon 2, which is dispensable for the activation of MAPK. Tat exon 2 has been shown to have a favorable effect on HIV-1 replication in tissue culture. Thus, Tat exon 2 plays an important role in HIV-1 replication. There is an important role of Tat exon 2 in $\mathrm{NF}_{-\mathrm{k}} \mathrm{B}$ activation and LTR mediated transcription. It was observed that $\mathrm{NF}_{-\mathrm{k}} \mathrm{B}$-dependent control of HIV-1 transcription by Tat exon 2 in T cells, results in enhanced viral replication in primary PBLs.

\section{CONCLUSION}

Human Immunodeficiency Virus type 1 (HIV-1) displays extraordinary genetic variation, leading to the classification of the viral strains into phylogenetically distinct groups and subtypes. Of the various subtypes of HIV-1, subtype C is linked to $\sim 50 \%$ of the infections globally and is associated with rapidly growing epidemics in sub-Saharan Africa and parts of Asia, including India and China. In addition to genetic and demographic factors, biological properties unique to the subtype $\mathrm{C}$ viruses may also play a role in their exponential proliferation. Precisely it is not known that 
how Tat exhibits differential pathogenic properties. HIV-1 Tat protein is indispensable for HIV/AIDS pathogenesis and progression because of its transactivation functions and extracellular transmodulatory functions. The Tat protein undergoes multiple posttranslational modifications, such as acetylation, ubiquitination and methylamine, which are responsible for the regulation of its interaction rather with RNA or/and cellular factors like cyclin-T1, P300 /CBP and PCAF. Several Analytical studies show that most of the target residues of Tat protein, for posttranslation modification are conserved among the amino acids sequences of different and variety of patients. HIV-1 C Tat protein may efficient in transactivation because it shows stable interaction with $\mathrm{pTEFb}$ to elongate transcription of viral genome. The HIV Tat protein is basically a transactivator protein to elevate the transcription of viral genome to produce more virions. HIV Tat plays extracellular role by mimicking chemokines illustrated the genetic and functional qualities of Tat. Tat exon 1 is moderately conserved and Tat exon 2 has highly conserved motifs. Therefore, tat gene can certainly be targeted against the combat between human and HIV. It has been reported that endolysosomes actively involved in the HIV-1 Tat-induced neurotoxicity and henceforth may definitely act as a target for therapeutic intervention against HAND (Hui et al., 2012).

The Tat protein based vaccine approach is important because it plays a crucial role in HIV-1 replication and it helps virus to be more pathogenic through its multifaceted functions and activities. Extracellular Tat also interacts with a variety of surface receptors, including integrin receptors and members of vascular endothelial growth factors family and activates various intracellular transduction pathways. Evidence also indicates that Tat can activate the expression of a number of heterologous viral promoters, such as herpes viruses, HTLV-1 and -2 and others, implying that Tat may be also involved in the development of AIDSassociated opportunistic infections. Finally, the Tat protein, both intracellular and extracellular, exerts key roles in the pathogenesis of AIDS-associated tumors and HIV associated Dementia described as earlier.

Focusing on the Tat protein represents a radically different approach to construct a vaccine against HIV-1. The rationale for this approach is based upon the following scientific evidences. Tat can be well targeted as it is proved that Antibody to HIV-1 Tat protein inhibits the replication of virus in culture (Steinaa et al., 1994). It is also scientifically believed that HIV-1 Tat protein can act both as a target and a tool in therapeutic approaches against HIV virus (Watson and Edwards, 1999).
Tat antibodies in human serum: Tat based vaccine creates interest, by the discovery that seropositive long term non progressors had a higher level of Tat antibodies than rapid progressors. So therefore it is a notable thing that mutation in Tat variants can affect immunogenicity and recognition.

The presence of anti-Tat antibodies seems to protect infected individuals from progressing to AIDS. In fact, anti-Tat antibodies are more frequently found in the early stage of the disease than during the symptomatic stages. Finally, in studies conducted over long period of times (longitudinal studies), anti-Tat antibodies have been associated with a slower disease progression.

Anti-Tat cytotoxic (CD8+) T Lymphocytes (CTLs) are frequently found in individuals who are infected naturally by HIV. CD8+T cell responses correlate with early virus control in both humans and experimental animals.

The immunogenic regions (those capable of producing an immune response) of Tat are conserved among the different HIV-1 clades, different evolutionary forms of the HIV-1 virus that have originated over the years. In fact, cross-clade recognition of the Tat $B$ clade has been observed with sera from Ugandan, South African and Italian patients with infections of different subtypes. In addition, key regions of the Tat protein are well conserved among the circulating virus clades. The data from these studies suggests that a Tat vaccine may be useful in different geographic areas of the world.

In addition to representing a valuable antigen for an HIV/AIDS vaccine, biologically active Tat has immunomodulatory features that make it an attractive adjuvant (substance that increases the immune response) for other antigens. Recent data shows that Monocyte-Derived Dendritic Cells (MDDC) and macrophages efficiently and rapidly take up native Tat, i.e., the biologically active form of Tat. After uptake, native Tat promotes MDDC maturation and activation. Activation includes increased expression of $\mathrm{MHC}$ antigens and co-stimulatory molecules, as well as increased production of cytokines and chemokines in TH-1 (helper T cells). All these events are key to obtain a successful iummunogenic response to vaccination.

The RGD motif of Tat exon 2 has a crucial role in cell adhesion and it is quite conserved also, so it may definitely serve as a drug target.

The ESKKKVE motif is also highly conserved and important motif of Tat exon 2, which has a vital role in viral transcription, henceforth vaccine targeting this motif can be genuinely though of and deviced.

The data suggests that vaccination with Tat may modify the virus-host dynamics and control HIV-1 replication both in primary infection (preventive 
strategy) and in infected individuals (therapeutic strategy). Moreover, Tat can function as both an antigen and a potent adjuvant. Together, these features make Tat an optimal candidate for an HIV vaccine, alone or in combination with other antigens. Used in a therapeutic strategy, vaccinating seropositive (HIV-positive) patients with Tat should reduce HIV-1 replication and slow disease progression. Used in a preventive strategy, a Tatbased vaccine could modify the virus-host dynamics at the very beginning of HIV infection, should it occur. As a result, critical immune cells would not be depleted and progression of the infection would be interrupted. This scenario is based on accumulating evidence suggesting that the viral load at the beginning of the infection is a strong indicator of progression to disease. Another advantage of a Tat-based vaccine approach is that it would allow discriminating vaccinated from unvaccinated individuals. This is possible because a Tatbased vaccine does not contain structural HIV proteins on which current blood tests are based and therefore the Tat-vaccinated volunteer will not seroconvert (false positives). Italy has deviced the vaccine and send it for vaccine trials, same effort is required for the country to device for gene based drugs. Recently single immunization with the DermaVir therapeutic vaccine was tested safe and immunogenic in HIV-infected individuals by conducting experiments on potent induction of Tat-specific memory $\mathrm{T}$ cells, it was we speculated that DermaVir boosts $\mathrm{T}$ cell responses (Lisziewicz et al., 2012). HIV-1 Tat has been recognized as a striking target for both therapeutic and preventative vaccine against HIV-1. The Tat C-terminal region is of significant importance and manually designed Tat can present designed immunogenicity that upgrades Tat-specific antibodies against C-terminal regions (Liao et al., 2012).

Future prospective: The cationic Tat peptide (48-56 aa), called the Protein Transduction Domain (PTD) or Cell Penetrating Peptide, is widely used for intracellular delivery of macromolecules including oligonucleotides, peptides or proteins, low molecular weight drugs, nanoparticles and liposomes. This approach has also been exploited for developing novel neuroprotective strategies.

Need of the hour: Ever since the virus is been available to human knowledge, the mankind us running in the race against the virus, but this combat against this deadly virus is not that easy. Today we need gene specific targeting drugs, which can focus on one particular gene or a part of gene that is motif, which is conserved and is most stable. This stable part can be very well targeted by the designed drugs. This is not a single man's job; this requires a symphonic effect from researchers, scientists, drug designers, bioinformaticians, clinical researchers and policy makers. The need of the hour is to device a solid virus specific drug which may help mankind in combat with the virus.

\section{ACKNOWLEDGMENT}

Reachers are grateful to Council of Scientific and Industrial Research (CSIR-CCMB) India and $\mathrm{NIH}$ Awards (R37DA025576; R01MH085259) for the encouragement and support for this study.

\section{REFERENCES}

Bartz, S.R. and M. Emerman, 1999. Human immunodeficiency virus type 1 Tat induces apoptosis and increases sensitivity to apoptotic signals by up-regulating FLICE/caspase-8. J. Virol., 73: 1956-1963 PMID: 9971775

Brake, D.A., C. Debouck and G. Biesecker, 1990. Identification of an Arg-Gly-Asp (RGD) cell adhesion site in human immunodeficiency virus type 1 transactivation protein, tat. J. Cell. Biol., 111: 1275-1281. PMID: 2202737

Buonaguro, L., G. Barillari, H.K. Chang, C.A. Bohan and V. Kao et al., 1992. Effects of the human immunodeficiency virus type 1 Tat protein on the expression of inflammatory cytokines. J. Virol., 66: 7159-7167. PMID: 1279199

Charnay, N., R. Ivanyi-Nagy, R. Soto-Rifo, T. Ohlmann and M. López-Lastra et al., 2009. Mechanism of HIV-1 Tat RNA translation and its activation by the Tat protein. Retrovirology, 6: 74 PMID: 19671151

Courter, D., H. Cao, S. Kwok, C. Kong and A. Banh et al., 2010. The RGD domain of human osteopontin promotes tumor growth and metastasis through activation of survival pathways. PLoS One, 5: e9633. PMID: 20224789

Czudnochowski, N., C.A. Bösken and M. Geyer, 2012. Serine-7 but not serine-5 phosphorylation primes RNA polymerase II CTD for P-TEFb recognition. Nat. Commun., 15: 842-842. PMID: 22588304

D'Aversa, T.G., K.O. Yu and J.W. Berman, 2004. Expression of chemokines by human fetal microglia after treatment with the human immunodeficiency virus type 1 protein Tat. J. Neur., 10: 86-97. PMID: 15204927

Debaisieux, S., F. Rayne, H. Yezid and B. Beaumelle, 2012. The ins and outs of HIV-1 Tat. Traffic., 13: 355-363. PMID: 21951552 
Derse, D., M. Carvalho, R. Carroll and B.M. Peterlin, 1991. A minimal lentivirus tat. J. Virol., 65: 70127015. PMID: 1658392

Dey, S.S., Y. Xue, M.P. Joachimiak, G.D. Friedland and J.C. Burnett et al., 2012. Mutual information analysis reveals coevolving residues in Tat that compensate for two distinct functions in HIV-1 gene expression. J. Biol. Chem., 287: 7945-7955 PMID: 22253435

Douglas, J.L., J.K. Gustin, B. Dezube, J.L. Pantanowitz and A.V. Moses, 2007. Kaposi's sarcoma: A model of both malignancy and chronic inflammation. Panminerva Med., 49: 119-138. PMID: 17912148

El-Hage, N., G. Wu, J. Wang, J. Ambati and P.E. Knapp et al., 2006. HIV-1 Tat and opiate-induced changes in astrocytes promote chemotaxis of microglia through the expression of MCP-1 and alternative chemokines. Glia, 53: 132-146. PMID: 16206161

Ensoli, B., S.Z. Salahuddin and R.C. Gallo, 1989. AIDS-associated Kaposi's sarcoma: A molecular model for its pathogenesis. Cancer Cells, 1: 93-96. PMID: 2561910

Eugenin, E.A., G. Dyer, T.M. Calderon and J.W. Berman, 2005. HIV-1 tat protein induces a migratory phenotype in human fetal microglia by a CCL2 (MCP-1)-dependent mechanism: Possible role in NeuroAIDS. Glia, 49: 501-510. PMID: 15578658

Frankel, A.D., L. Chen, R.J. Cotter and C.O. Pabo, 1988. Dimerization of the tat protein from human immunodeficiency virus: A cysteine-rich peptide mimics the normal metal-linked dimer interface. Proc. Natl. Acad. Sci. USA, 85: 6297-6300. PMID: 2842763

Gonzalez-Hernandez, M.J., M.D. Swanson, R. Contreras-Galindo, S. Cookinham and S.R. King et al., 2012. Expression of human endogenous retrovirus type $\mathrm{K}$ (HML-2) Is activated by the tat protein of HIV-1. J. Virol., 86: 7790-7805. PMID: 22593154

Graeble, M.A., M.J. Churcher, A.D. Lowe, M.J. Gait and J. Karn, 1993. Human immunodeficiency virus type 1 transactivator protein, tat, stimulates transcriptional read-through of distal terminator sequences in vitro. Proc. Natl. Acad. Sci. USA., 90: 6184-6188. PMID: 8327498

Haughey, N.J., C.P. Holden, A. Nath and J.D. Geiger, 1999. Involvement of inositol 1,4,5-trisphosphateregulated stores of intracellular calcium in calcium dysregulation and neuron cell death caused by HIV-1 protein tat. J. Neurochem.73: 1363-1374. PMID: 10501179
Herce, H.D. and A.E. Garcia, 2007. Molecular dynamics simulations suggest a mechanism for translocation of the HIV-1 TAT peptide across lipid membranes. Proc. Natl. Acad. Sci. USA., 104: 20805-20810 PMID: 18093956

Howcroft, T.K., K. Strebel, M.A. Martin and D.S. Singer, 1993. Repression of MHC class I gene promoter activity by two-exon tat of HIV. Science, 260: 1320-1322. PMID: 8493575

Hui, L., X. Chen, N.J. Haughey and J.D. Geiger, 2012. Role of endolysosomes in HIV-1 Tat-induced neurotoxicity. ASN Neuro., 4: e00091-e00091. PMID: 22591512

Izmailova, E., F.M. Bertley, Q. Huang, N. Makori and C.J. Miller et al., 2003. HIV-1 Tat reprograms immature dendritic cells to express chemoattractants for activated $\mathrm{T}$ cells and macrophages. Nat. Med., 9: 191-197. PMID: 12539042

Jager, S., P. Cimermancic, N. Gulbahce, J.R. Johnson and K.E. McGovern et al., 2011. Global landscape of HIV-human protein complexes. Nature, 481: 365-370. PMID: 22190034

Kessler, M. and M.B. Mathews, 1992. Premature termination and processing of human immunodeficiency virus type 1-promoted transcripts. J. Virol., 66: 4488-4496. PMID: 1602555

Koken, S.E., A.E. Greijer, K. Verhoef, J. van Wamel and A.G. Bukrinskaya et al., 1994. Intracellular analysis of in vitro modified HIV tat protein. J. Biol. Chem., 269: 8366-8375. PMID: 8132560

Kolson, D.L., J. Buchhalter, R. Collman, B. Hellmig and C.F. Farrell et al., 1993. HIV-1 Tat alters normal organization of neurons and astrocytes in primary rodent brain cell cultures: RGD sequence dependence. AIDS Res. Hum. Retroviruses, 9: 677-685. PMID: 8369172

Kruman, I.I., A. Nath and M.P. Mattson, 1998. HIV-1 protein Tat induces apoptosis of hippocampal neurons by a mechanism involving caspase activation, calcium overload and oxidative stress. Exp. Neurol., 154: 276-288. PMID: 9878167

Kuppuswamy, M., T. Subramanian, A. Srinivasan and G. Chinnadurai, 1989. Multiple functional domains of Tat, the trans-activator of HIV-1, defined by mutational analysis. Nucl. Acids Res., 17: 35513561. PMID: 2542902

Li, C.J., Y. Ueda, B. Shi, L. Borodyansky and L. Huang et al., 1997. Tat protein induces self-perpetuating permissivity for productive HIV-1 infection. Proc. Natl. Acad. Sci. USA , 94: 8116-8120. PMID: 9223324 
Li, W., G. Li, J. Steiner and A. Nath, 2009. Role of Tat protein in HIV neuropathogenesis. Neurotox. Res., 16: 205-220. PMID: 19526283

Li, W., Y. Huang, R. Reid, J. Steiner and T. MalpicaLlanos et al., 2008. NMDA receptor activation by HIV-tat protein is clade dependent. J. Neu., 28: 12190-12198. PMID: 19020013

Liao, W., Q. Chen, J. Cao, G. Tan and Z. Zhu et al., 2012. A designed tat immunogen generates enhanced anti-Tat C-terminal antibodies. Vaccine, 30: 2453-2461. PMID: 22330127

Lisziewicz, J., N. Bakare, S.A. Calarota, D. Bánhegyi and J. Szlávik et al., 2012. Single DermaVir Immunization: Dose-Dependent Expansion of Precursor/Memory T Cells against All HIV Antigens in HIV-1 Infected Individuals. PLoS One. 7: e35416. PMID: 22590502

López-Huertas, M.R., S. Callejas, D. Abia, E. Mateos and A. Dopazo et al., 2010. Modifications in host cell cytoskeleton structure and function mediated by intracellular HIV-1 Tat protein are greatly dependent on the second coding exon. Nucleic Acids Res., 38: 3287-3307. PMID: 20139419

Ma, M. and A. Nath, 1997. Molecular determinants for cellular uptake of Tat protein of human immunodeficiency virus type 1 in brain cells. J. Virol., 71: 2495-2499. PMID: 9032389

Mahlknecht, U., I. Dichamp, A. Varin, C. Van Lint and G. Herbein, 2008. NF-kappaB-dependent control of HIV-1 transcription by the second coding exon of Tat in T cells. J. Leukoc. Biol., 83: 718-727. PMID: 18070983

Mayol, K., S. Munier, A. Beck, B. Verrier and C. Guillon, 2007. Design and characterization of an HIV-1 Tat mutant: Inactivation of viral and cellular functions but not antigenicity. Vaccine, 25: 60476060. PMID: 17604883

Nath, A., K. Psooy, C. Martin, B. Knudsen and D.S. Magnuson et al., 1996. Identification of a human immunodeficiency virus type 1 Tat epitope that is neuroexcitatory and neurotoxic. J. Virol., 70: 14751480. PMID: 8627665

Neuveut, C., R.M. Scoggins, D. Camerini, R.B. Markham and K.T. Jeang, 2003. Requirement for the second coding exon of Tat in the optimal replication of macrophage-tropic HIV-1. J. Biomed. Sci., 10: 651-660. PMID: 14576468

New, D.R., M. Ma, L.G. Epstein, A. Nath and H.A. Gelbard, 1997. Human immunodeficiency virus type 1 Tat protein induces death by apoptosis in primary human neuron cultures. J. Neur., 3: 168173. PMID: 9111179
Norman, J.P., S.W. Perry, K.A. Kasischke, D.J. Volsky and H.A. Gelbard, 2007. HIV-1 trans activator of transcription protein elicits mitochondrial hyperpolarization and respiratory deficit, with dysregulation of complex IV and nicotinamide adenine dinucleotide homeostasis in cortical neurons. J. Immunol., 178: 869-876. PMID: 17202348

Pulliam, L., B. Sun, H. Rempel, P.M. Martinez and J.D. Hoekman et al., 2007. Intranasal tat alters gene expression in the mouse brain. J. Neur. Pharmacol., 2: 87-92. PMID: 18040830

Ranga, U., R. Shankarappa, N.B. Siddappa, L. Ramakrishna and R. Nagendran et al., 2004. Tat protein of human immunodeficiency virus type 1 subtype $\mathrm{C}$ strains is a defective chemokine. J. Virol., 78: 2586-2590. PMID: 14963162

Ruben, S., A. Perkins, R. Purcell, K. Joung and R. Sia et al., 1989. Structural and functional characterization of human immunodeficiency virus tat protein. J. Virol., 63: 1-8. PMID: 2535718

Ruoslahti, E., 1996. RGD and other recognition sequences for integrins. Annu. Rev. Cell. Dev. Biol., 12: 697-715. PMID: 8970741

Schiralli, L.G.M. and A.J. Henderson, 2012. Mechanisms of HIV transcriptional regulation and their contribution to latency. Mol. Biol. Int. 2012: 614120-614120. PMID: 22701796

Stauber, R.H. and G.N. Pavlakis, 1998. Intracellular trafficking and interactions of the HIV-1 Tat protein. Virology. 252: 126-136. PMID: 9875323

Steinaa, L., A.M. Sørensen, J.O. Nielsen and J.E. Hansen, 1994. Antibody to HIV-1 Tat protein inhibits the replication of virus in culture. Arch. Virol., 139: 263-271. PMID: 7832634

Tahirov, T.H., N.D. Babayeva, K. Varzavand, J.J. Cooper and S.C. Sedore et al., 2010. Crystal structure of HIV-1 Tat complexed with human PTEFb. Nature, 465: 747-751. PMID: 20535204

Verhoef, K., A. Klein and B. Berkhout, 1996. Paracrine activation of the HIV-1 LTR promoter by the viral Tat protein is mechanistically similar to transactivation within a cell. Virology, 225: 316-327. PMID: 8918918

Vogel, B.E., S.J. Lee, A. Hildebrand, W. Craig and M.D. Pierschbacher et al., 1993. A novel integrin specificity exemplified by binding of the alpha $\mathrm{v}$ beta 5 integrin to the basic domain of the HIV Tat protein and vitronectin. J. Cell Biol., 121: 461-468. PMID: 7682219 
Watson, K. and R.J. Edwards, 1999. HIV-1-transactivating (Tat) protein: Both a target and a tool in therapeutic approaches. Biochem. Pharmacol., 58: 1521-1528. PMID: 10535742

Westendorp, M.O., M. Li-Weber, R.W. Frank and P.H. Krammer, 1994. Human immunodeficiency virus type 1 Tat upregulates interleukin-2 secretion in activated T cells. J. Virol, 68: 4177-4185. PMID: 8207793

Westendorp, M.O., R. Frank, C. Ochsenbauer, K. Stricker and J. Dhein et al., 1995. Sensitization of T cells to CD95-mediated apoptosis by HIV-1 Tat and gp120. Nature, 375: 497-500. PMID: 7539892

Wires, E.S., D. Alvarez, C. Dobrowolski, Y. Wang and M. Morales et al., 2012. Methamphetamine activates nuclear factor kappa-light-chain-enhancer of activated B cells (NF- $\kappa \mathrm{B}$ ) and induces Human Immunodeficiency Virus (HIV) transcription in human microglial cells. J. Neurovirol., PMID: 22618514
Xiao, H., C. Neuveut, M. Benkirane and K.T. Jeang, 1998. Interaction of the second coding exon of Tat with human EF-1 delta delineates a mechanism for HIV-1-mediated shut-off of host mRNA translation. Biochem. Biophys. Res. Commun., 244: 384-389. PMID: 9514931

Zauli, G., D. Gibellini, A. Caputo, A. Bassini and M. Negrini et al., 1995. The human immunodeficiency virus type-1 Tat protein upregulates Bcl-2 gene expression in Jurkat T-cell lines and primary peripheral blood mononuclear cells. Blood, 86: 3823-3834. PMID: 7579350

Zhang, S.M., Y. Sun, R. Fan, Q.Z. Xu and X.D. Liu et al., 2010. HIV-1 Tat regulates cyclin B1 by promoting both expression and degradation. FASEB J., 2: 495-503. PMID: 19825974 